Revue des patrimoines

29 | 2016

Ensembles mobiliers, industriels, techniques.

Connaissance, protection, conservation, présentation au public

\title{
L'église Saint-Nicaise de Reims. Un ensemble décoratif authentique
}

The Saint-Nicaise Church at Reims. A genuine decorative ensemble

Isabelle Morin Loutrel

\section{OpenEdition}

Journals

Édition électronique

URL : http://journals.openedition.org/insitu/12995

DOI : 10.4000/insitu. 12995

ISSN : $1630-7305$

Éditeur

Ministère de la culture

Référence électronique

Isabelle Morin Loutrel, «L'église Saint-Nicaise de Reims. Un ensemble décoratif authentique », In Situ [En ligne], 29 | 2016, mis en ligne le 11 août 2016, consulté le 19 avril 2019. URL : http:// journals.openedition.org/insitu/12995 ; DOI : 10.4000/insitu.12995

Ce document a été généré automatiquement le 19 avril 2019

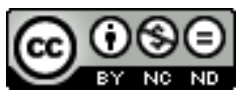

In Situ Revues des patrimoines est mis à disposition selon les termes de la licence Creative Commons Attribution - Pas d'Utilisation Commerciale - Pas de Modification 4.0 International. 


\title{
L'église Saint-Nicaise de Reims. Un ensemble décoratif authentique
}

The Saint-Nicaise Church at Reims. A genuine decorative ensemble

\author{
Isabelle Morin Loutrel
}

1 L'église Saint-Nicaise est emblématique pour la ville de Reims à deux titres au moins. Elle s'inscrit au cœur d'un programme immobilier social voulu par l'industriel Georges Charbonneaux (1865-1933) dès $1912^{1}$, elle reprend le vocable de la prestigieuse église abbatiale gothique Saint-Nicaise, située alors hors des remparts de la ville, abandonnée puis dépecée après 1793. Destinée à la population de familles nombreuses habitant la cité du Chemin Vert, elle fut très tôt l'objet d'un programme décoratif voulu par son commanditaire Georges Charbonneaux puis par l'architecte lui-même, Jacques-Marcel Auburtin (1872-1926) ${ }^{2}$ (fig. 1). 
Figure 1

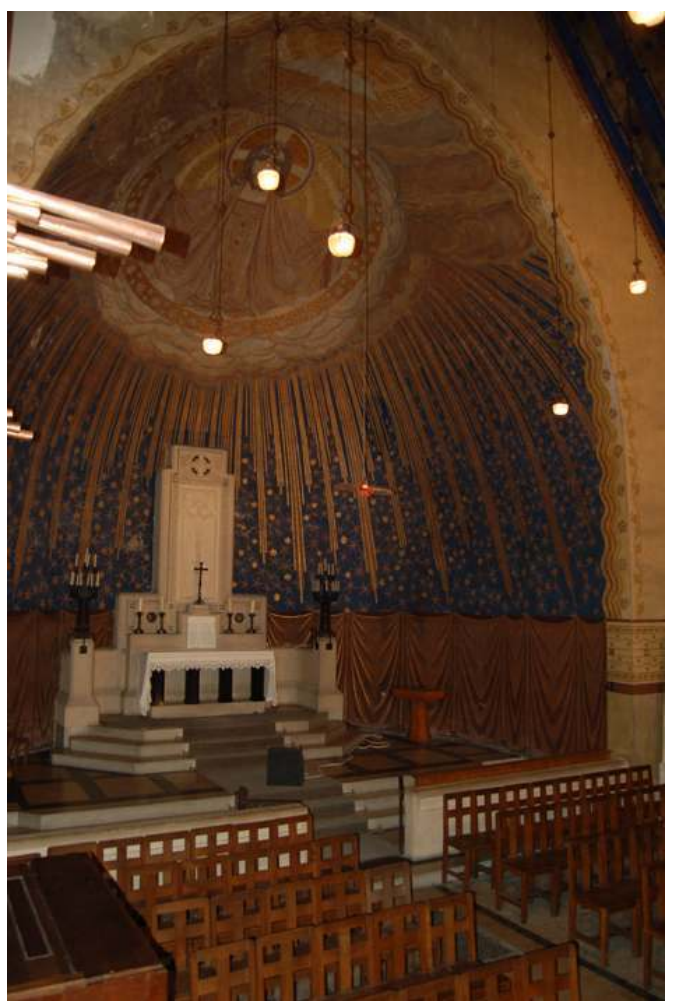

Intérieur de l'église Saint-Nicaise, vue vers le chœur. Peintures de Gustave Jaulmes, assisté d'Henri Menu, 1924.

Phot. CRMH Champagne-Ardenne. (c) CRMH Champagne-Ardenne.

Le terme de "programme " peut être utilisé ici dans la mesure où il y eut un dialogue constant entre le maître d'ouvrage et le maître d'œuvre pour orner l'église au fur et à mesure des idées et des opportunités de rencontres avec des artistes ${ }^{3}$. L'entregent de l'industriel fut décisif et son amitié pour Maurice Denis est sans doute à l'origine des nombreux autres contacts qu'il eut avec le monde de l'art. Comme souvent, c'est la volonté d'un homme qui permit une cohérence du propos ainsi qu'une continuité dans les travaux, aboutissant à une réalisation remarquable par son unité d'ensemble. Cette unité d'ensemble est encore aujourd'hui très authentique grâce au propriétaire du lieu, le Foyer Rémois, office d'habitation à loyers modérés, qui n'a pas cessé, depuis la création de la cité du Chemin Vert en 1920-1921, de favoriser et d'encourager la préservation des lieux. Ce cas de figure exceptionnel d'un fonctionnement sans faute jusqu'à nos jours, pour la mise en valeur et la conservation du bâti, est une réalité très positive pour les services des Monuments historiques qui ne sont finalement intervenus que tardivement dans la protection de l'église et de son mobilier". Aujourd'hui, une association très active, "Les amis de Saint-Nicaise du Chemin Vert ", favorise le lien avec la direction régionale des affaires culturelles en déchargeant le Foyer Rémois d'interventions peu en rapport avec sa mission première de gestion de logements sociaux. Elle a pour objectif de mieux faire connaître les lieux au grand public en le sensibilisant aux différentes créations artistiques qui ornent l'église, d'accompagner le propriétaire dans la sécurisation du bâtiment et dans la connaissance de son patrimoine mobilier ${ }^{5}$, de faire le lien avec les services de l'État et les associations patrimoniales pour la restauration de l'édifice. 
Figure 2

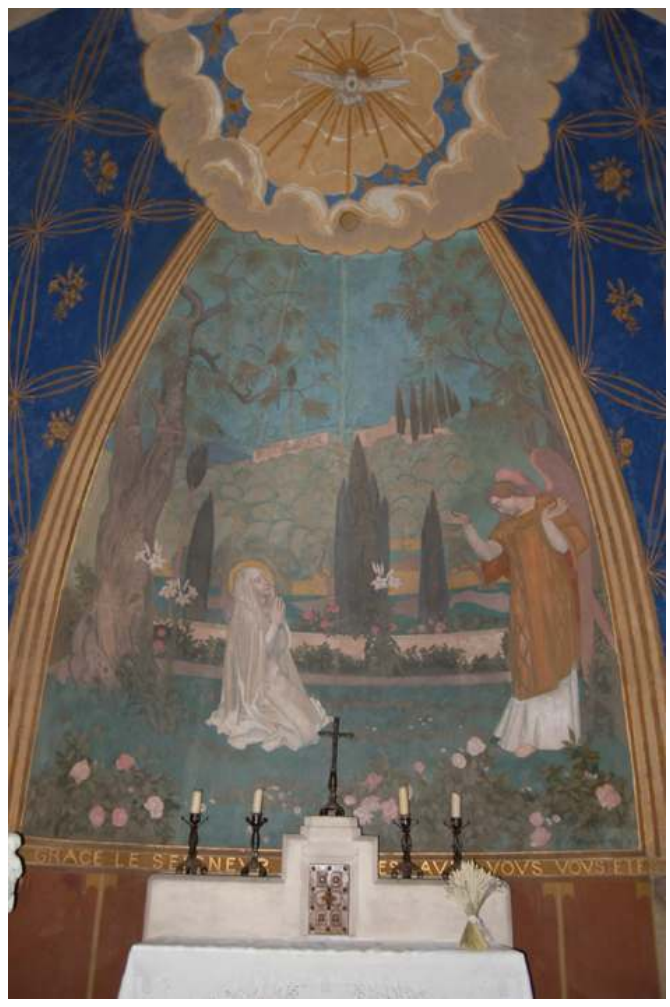

Chapelle latérale nord. Peinture de Maurice Denis, l'Annonciation, 1925-1926.

Phot. CRMH Champagne-Ardenne. (C) CRMH Champagne-Ardenne.

\section{Figure 3}

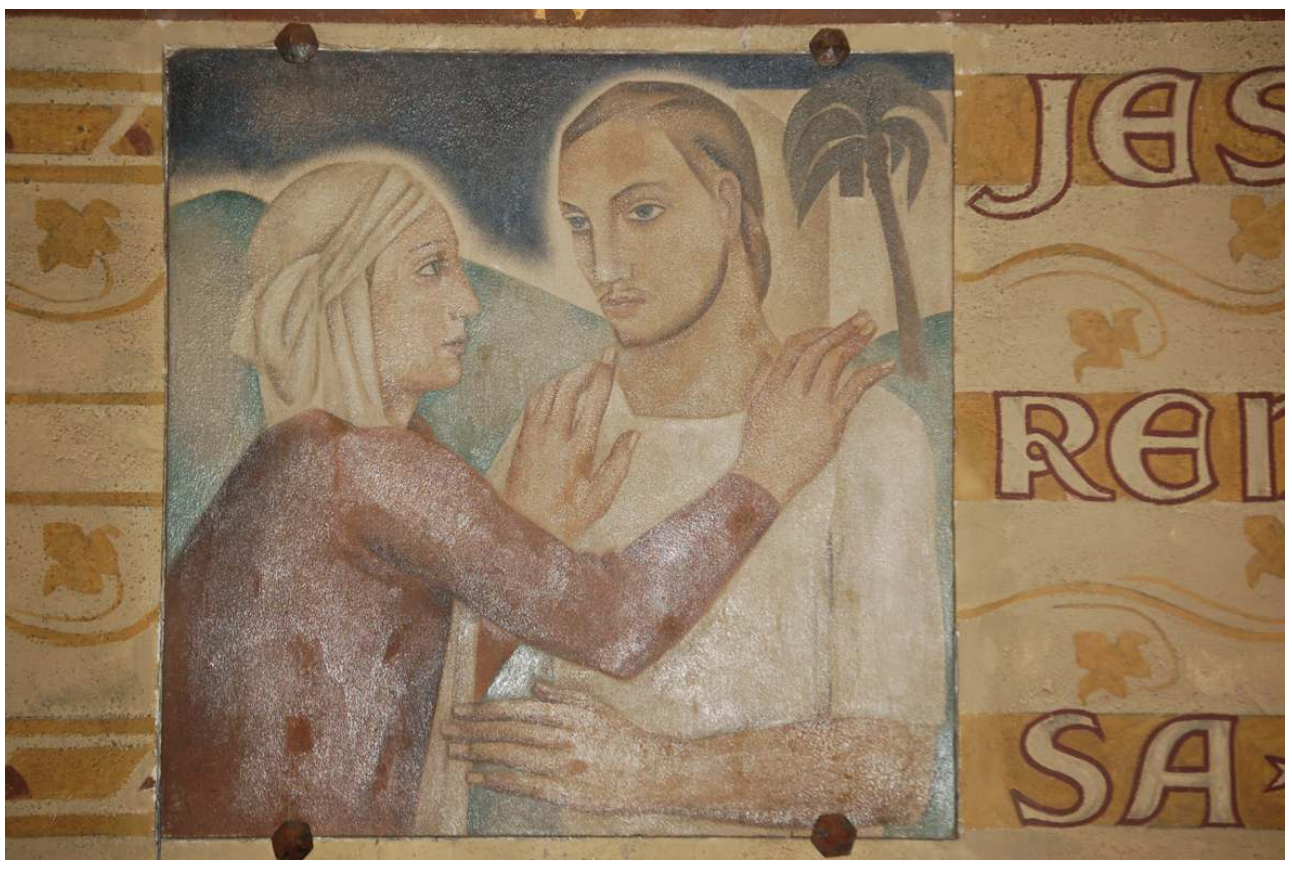

Chemin de Croix, quatrième station. Jean Berque, 1923-1924.

Phot. CRMH Champagne-Ardenne. (c) CRMH Champagne-Ardenne. 
Outre la qualité du décor pictural de l'église - peintures de Maurice Denis (1870-1943), de Gustave-Louis Jaulmes (1873-1959), de Jean Berque (1896-1954) (fig. 2, fig. 3) - et du décor sculpté - Emma Thiollier (1875-1973), Roger de Villiers (1887-1958) (fig. 4) ${ }^{6}$-, l'église Saint-Nicaise de Reims est également remarquable par son mobilier. Comme souvent dans les églises ou les propriétés privées, le maitre d'œuvre aime à dessiner le mobilier. C'est ici le cas, dans un contexte bien connu qui est celui de l'art décoratif, prenant en compte les moindres besoins de l'architecture fonctionnelle. C'est ainsi que Saint-Nicaise frappe d'entrée par l'harmonie du mobilier à usage religieux, autels, confessionnaux, chandeliers et grilles de communion. Sur le plan juridique, ces objets se partagent entre objets mobiliers (ou immeubles par destination) et immeubles par nature (peintures marouflées et autels) ${ }^{7}$. Ce sont des œuvres faisant partie intégrante de l'architecture pour le visiteur. À cela s'ajoutent le mobilier en bois, chaises et sièges des officiants $^{8}$. Même si l'attribution à l'architecte Auburtin de l'ensemble des chaises n'est pas confirmée par les documents (fig. 5) (il est de tradition de dire que la chaise de l'officiant fut réalisée plus récemment sur le modèle des autres), celles-ci fournissent un modèle homogène reprenant le motif de la croix que l'on peut retrouver, de façon sans doute allusive, sur les portes en chêne clôturant les différents espaces autour de la nef.

Figure 4

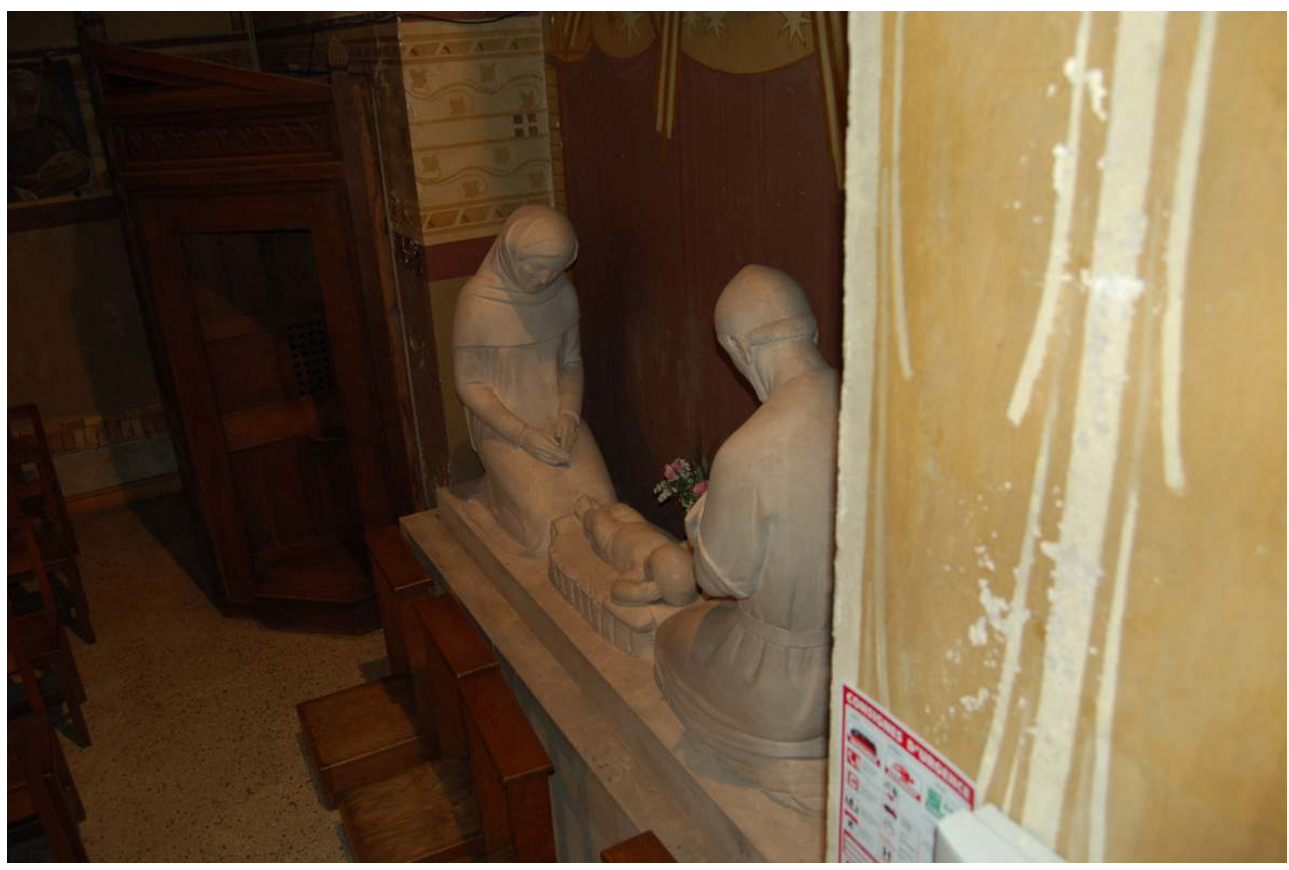

Nativité, chapelle nord, Roger de Villiers, 1925.

Phot. CRMH Champagne-Ardenne. ( C CRMH Champagne-Ardenne. 
Figure 5

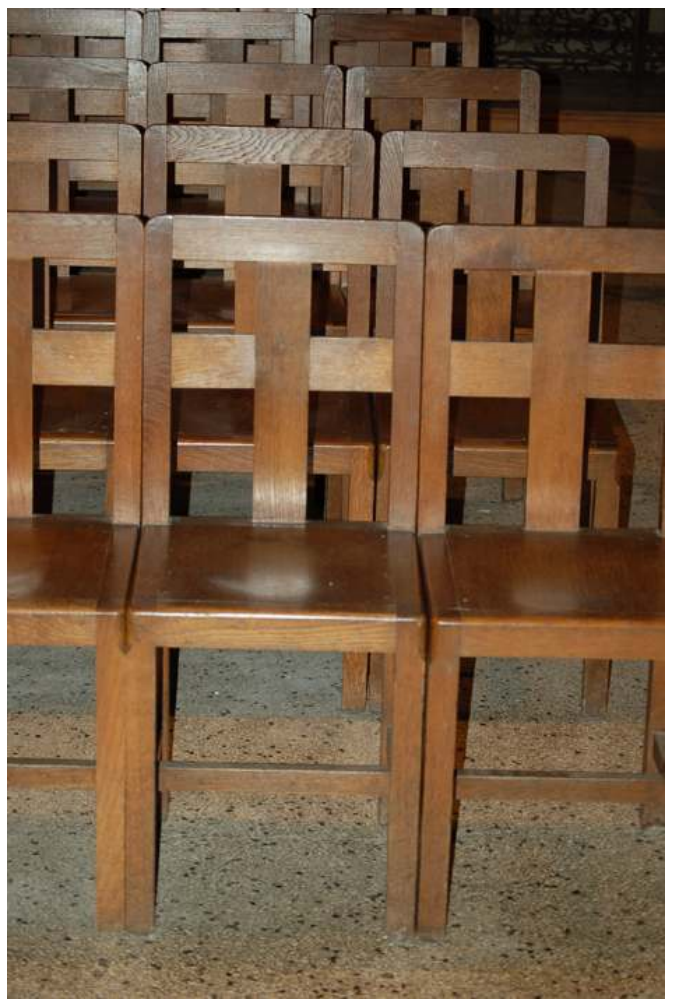

Chaises, Jean-Marcel Auburtin.

Phot. CRMH Champagne-Ardenne. (c) CRMH Champagne-Ardenne. 
Figure 6

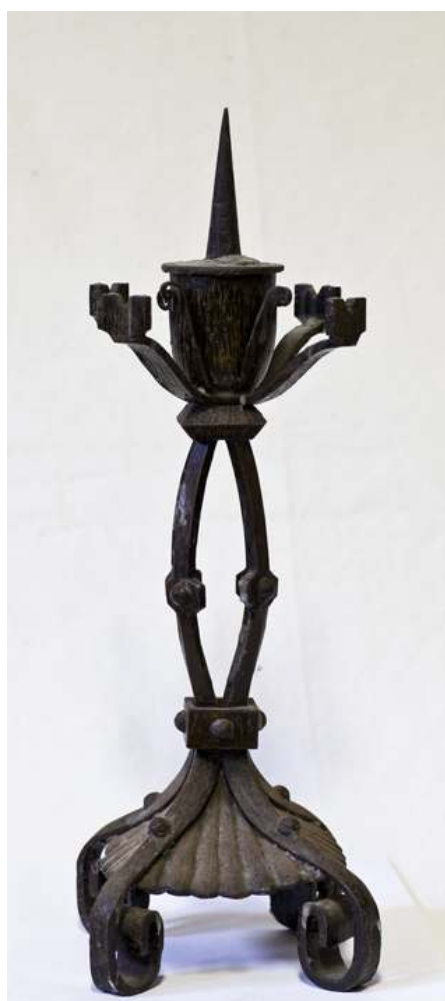

Porte-cierge, Jean-Marcel Auburtin et entreprise Berger-Bogart.

Phot. Potier, Dominique. (c) Dominique Potier.

\section{Figure 7}

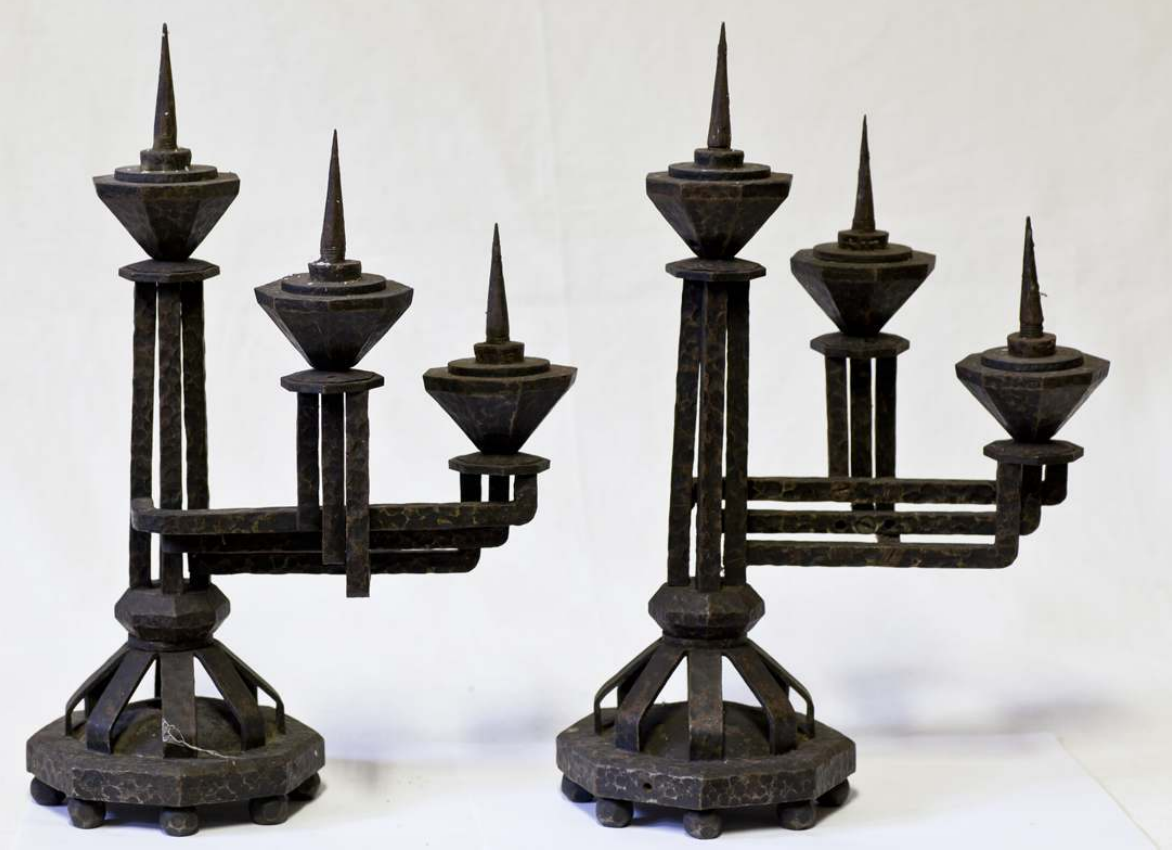

Chandelier à trois branches, Jean-Marcel Auburtin et entreprise Berger-Bogart.

Phot. Potier, Dominique. (C) Dominique Potier. 
4 Parmi le mobilier proprement liturgique, un ensemble de chandeliers et de porte-bougies, remarquables par leur matériau et leur facture ${ }^{9}$ orne les autels (fig. 6, fig. 7), eux-mêmes conçus avec tabernacle et niches clos par des ouvrants en verre et ferronnerie (fig. 8). Calices et ostensoir sont conservés dans la sacristie. Un remarquable ostensoir, en particulier, présente des formes Art déco qui font estimer sa datation à la même période que les objets mobiliers de l'église. Son étui porte la mention de l'entreprise d'orfèvrerie Poussielgue-Rusand (fig. 9) ${ }^{10}$. Quelques ornements liturgiques récents sont conservés.

Figure 8

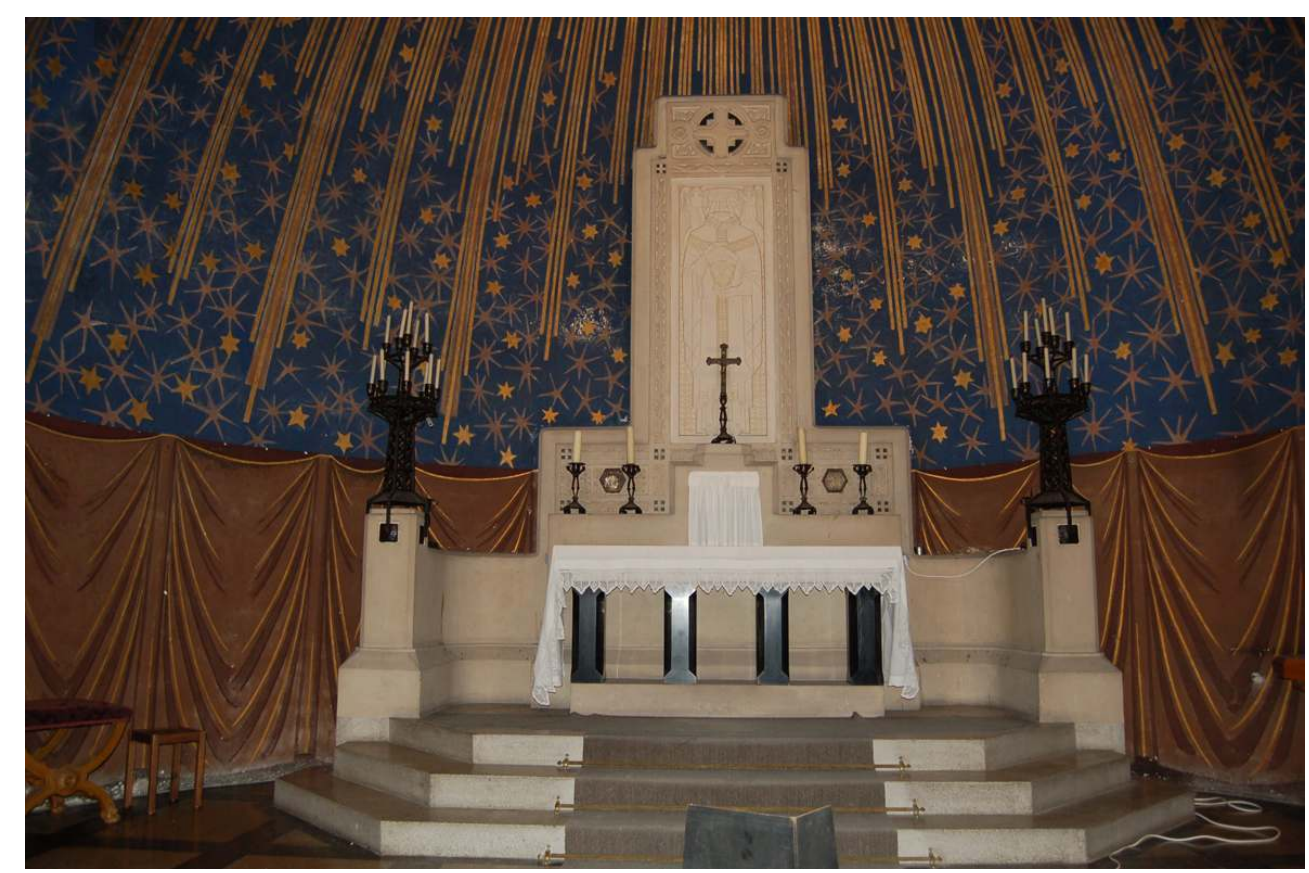

Autels et leur décor, Jean-Marcel Auburtin.

Phot. CRMH Champagne-Ardenne. (c) CRMH Champagne-Ardenne. 
Figure 9

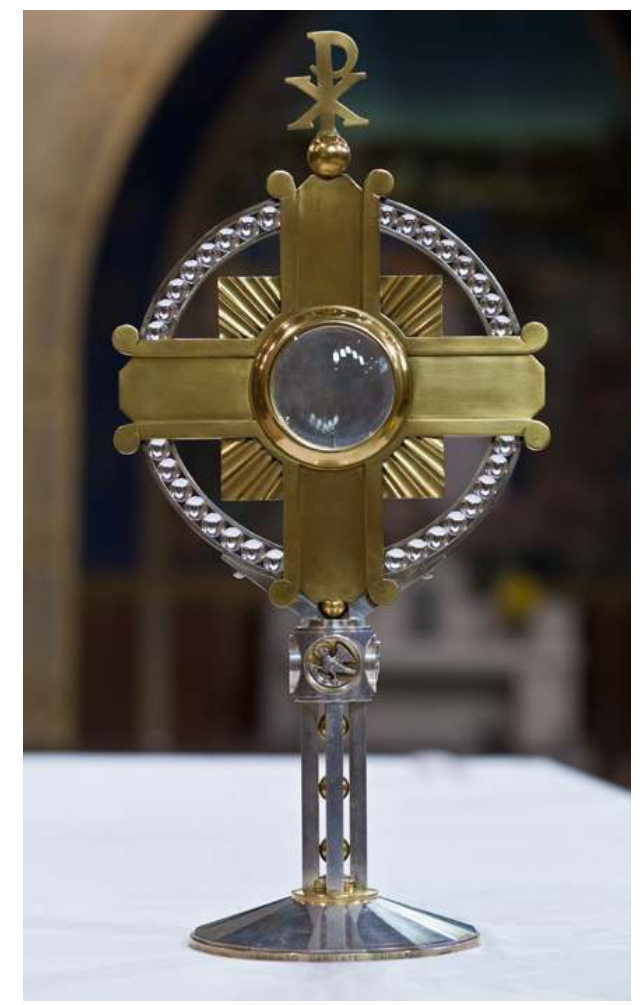

Ostensoir, laiton et argent.

Phot. Potier, Dominique. (c) Dominique Potier.

5 Cette description hâtive ne serait pas complète sans noter l'ensemble exceptionnel produit par René Lalique (1860-1945) comprenant les verrières, les luminaires et la colombe eucharistique.

6 Les verrières sont un prototype qui utilise la technique du coulé-moulé-pressé reprise ensuite dans d'autres lieux par l'artiste ${ }^{11}$. Elles ont été récemment déposées afin d'être restaurées car le temps a fait son effet sur les dalles (fig. 10). Tenant essentiellement par leur poids, les panneaux des baies se tassaient depuis plusieurs années, provoquant fissures et éclats dans le verre ${ }^{12}$. 
Figure 10

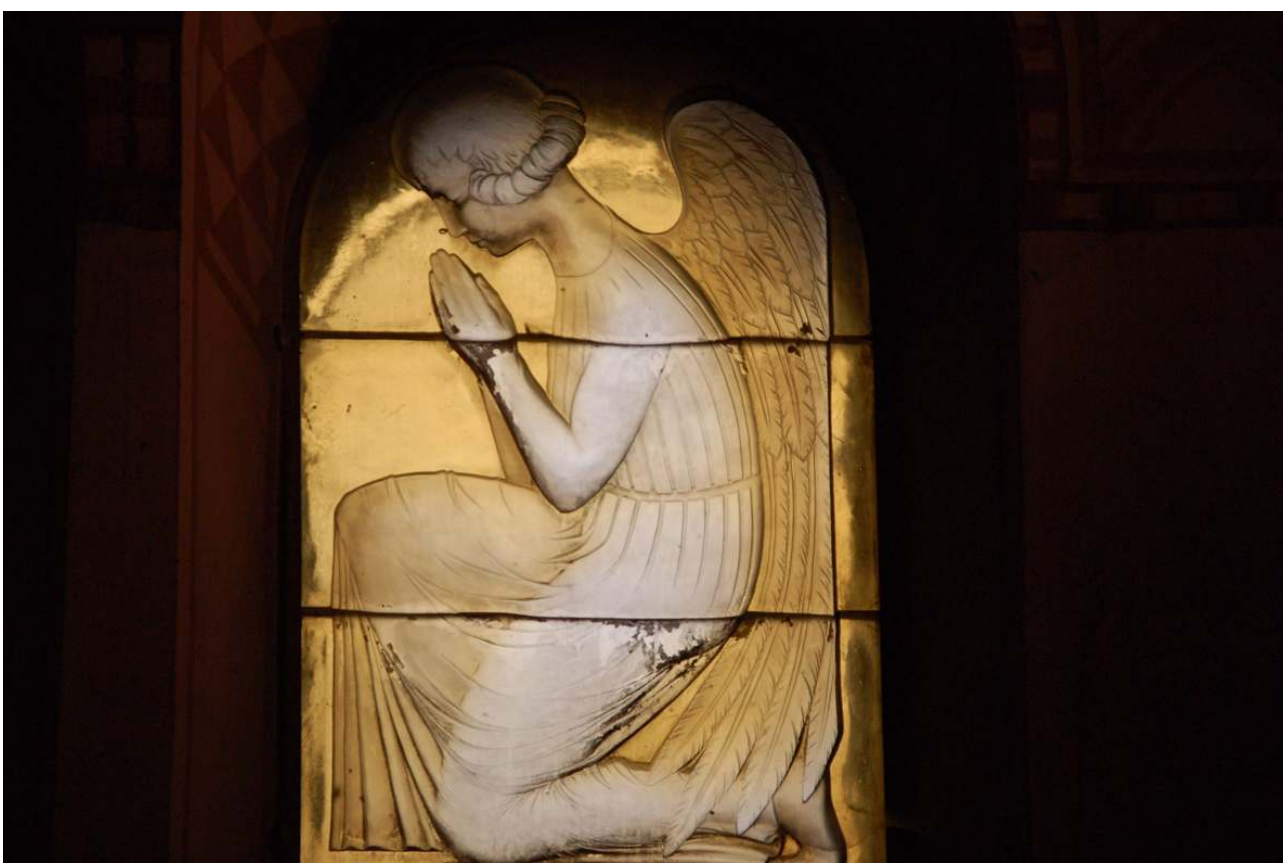

Verrières, détail, René Lalique, 1926.

Phot. CRMH Champagne-Ardenne. ( ) CRMH Champagne-Ardenne.

7 Les luminaires semblent visuellement aussi nombreux que les chaises et confèrent un effet à la fois moderne et singulier au lieu. Constitué de petits carrés de verre cruciformes maintenus chacun par une chaîne métallique, avec des agrafes de suspension formées de quatre petites croix dorées, leur présence crée un scintillement au même titre que l'emploi du doré dans les peintures ou le passage de la lumière au travers des anges des verrières. Ils participent de la « richesse » de la décoration, parfois critiquée par Maurice Denis lui-même (fig. 11, fig. 12). 
Figure 11

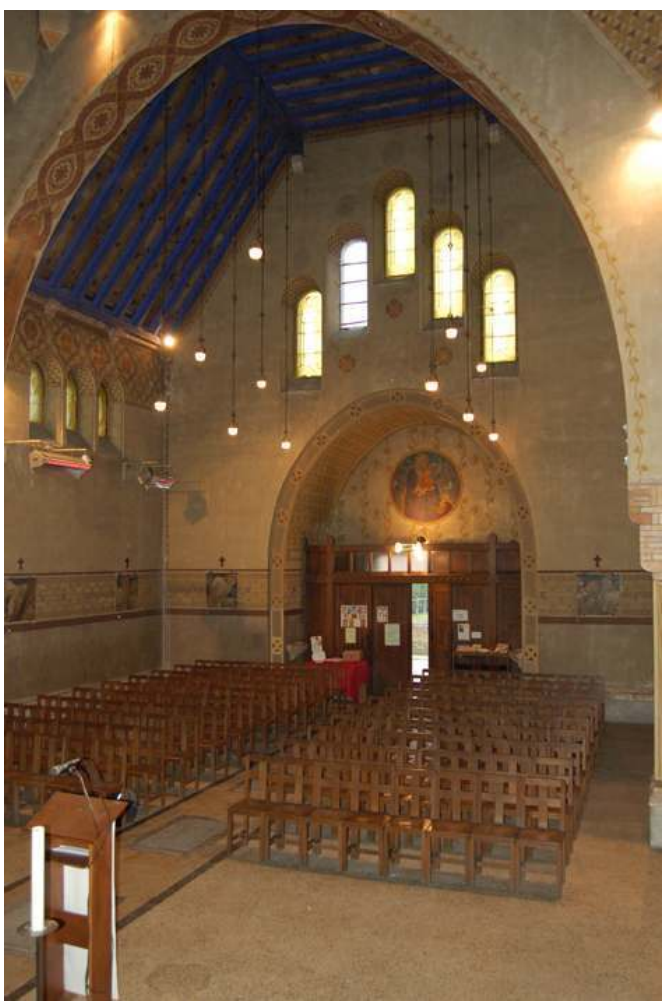

Luminaires, René Lalique.

Phot. CRMH Champagne-Ardenne. ( C CRMH Champagne-Ardenne. 
Figure 12

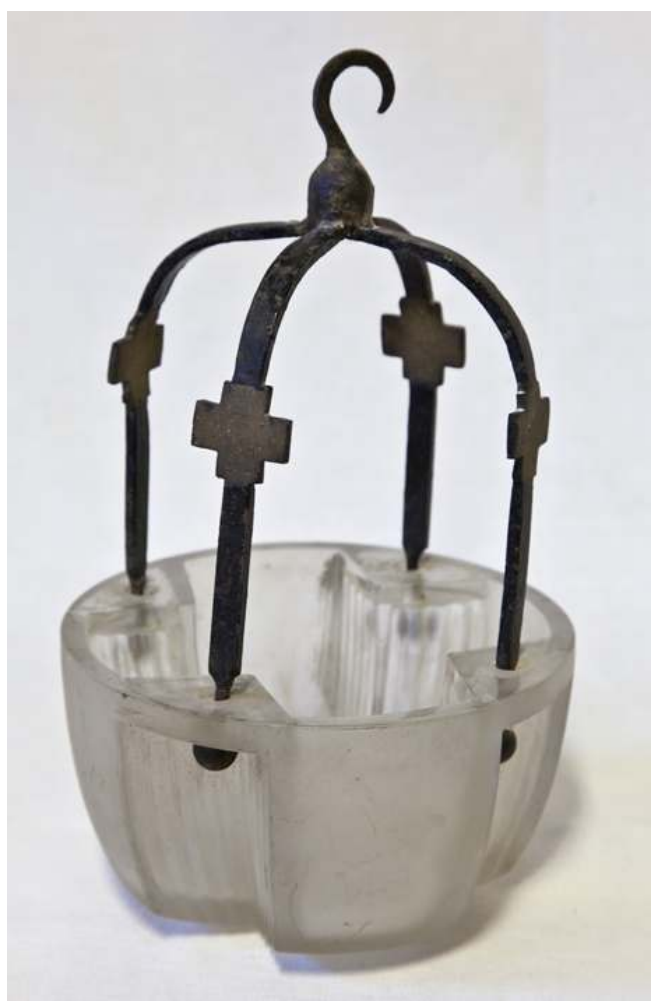

Luminaires, détail des suspensions.

Phot. Potier, Dominique. (c) Dominique Potier.

8 Le chef-d'œuvre de l'ensemble des suspensions est la colombe eucharistique, face à l'autel principal (fig. 13). 
Figure 13

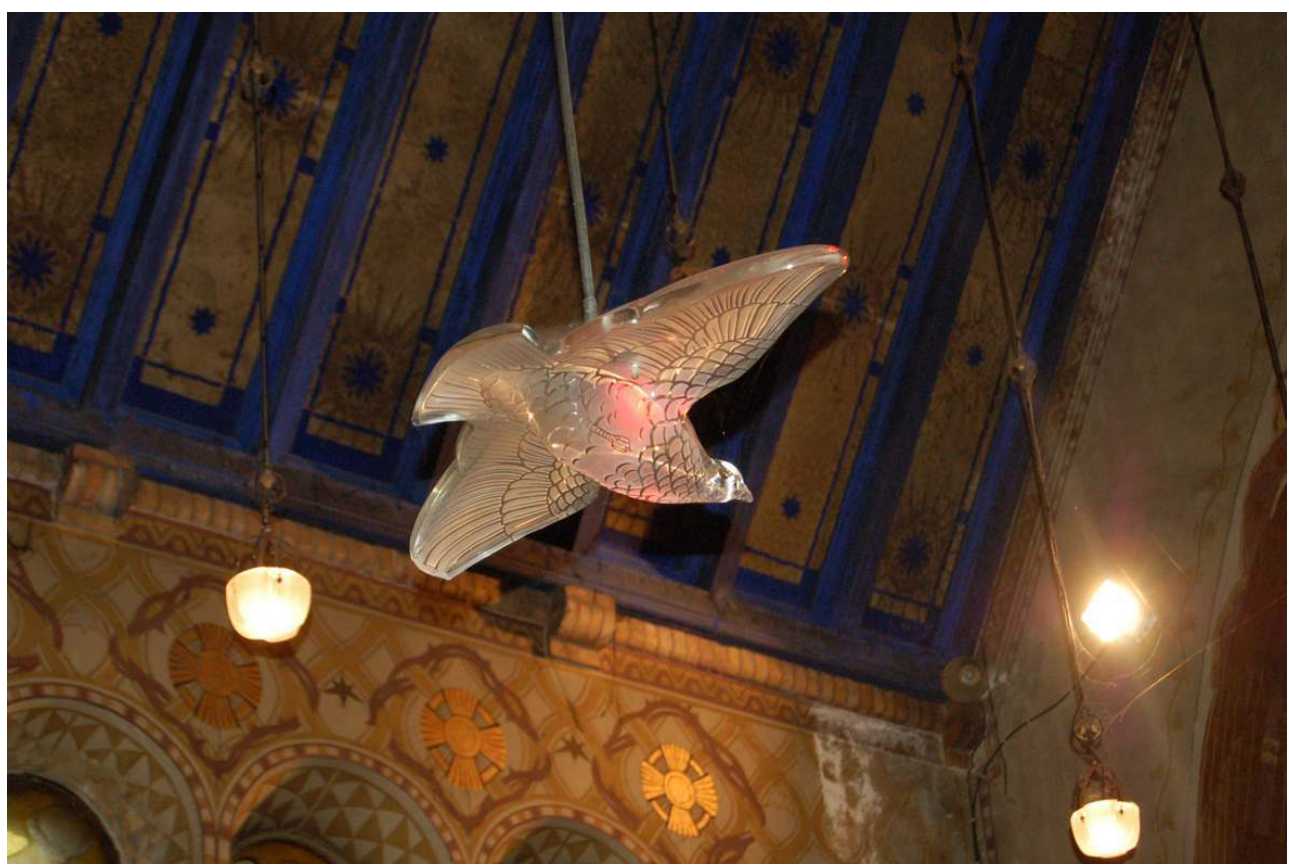

Colombe eucharistique, René Lalique.

Phot. CRMH Champagne-Ardenne. ( ) CRMH Champagne-Ardenne.

La spécificité de la cité du Chemin Vert et de l'église Saint-Nicaise en particulier est que la propriété du bâtiment, et du mobilier qu'il abrite, dépendait dès l'origine d'une société d'habitation à bon marché, le Foyer Rémois, qui confia la gestion de l'ensemble à l'association diocésaine de Reims par bail emphytéotique de 99 années, le $1^{\text {er }}$ juillet 1927. C'est dans ce but que fut établi devant notaire un acte indiquant le mode de propriété des lieux mais aussi et surtout, la liste des biens mobiliers de l'église. C'est aujourd'hui ce document qui constitue le point de départ de toute réflexion sur l'entretien et le maintien in situ des objets mobiliers de Saint-Nicaise ${ }^{13}$.

Les premiers termes de la « Désignation » de l'acte sont clairement exprimés dans le sens d'un tout:

«Cette église a reçu une décoration complète qui comprend notamment: ...» s'ensuit la liste des éléments de décor, mêlant peintures murales, peintures marouflées, vitraux et objets mobiliers stricto sensu. Les détails sont nombreux, en particulier ceux qui concernent les luminaires, les ferronneries, les sculptures (avec indication des matériaux, des formes et du nombre ditems), le baptistère et sa clôture, les éléments peints, la sacristie et son aménagement avec armoire et chasublier, l'orgue en cours de commande et sa tribune.

11 Dans les «Charges et conditions » du bail emphytéotique, les articles 2 et 3 stipulent : L'église Saint-Nicaise étant dotée d'une décoration et d'un mobilier cultuel qui doivent être considérés comme définitifs et complets, l'Association diocésaine ne pourra porter aucune atteinte au caractère architectural et décoratif de l'édifice dont l'aspect extérieur et les aménagements intérieurs ne pourront être modifiés. En conséquence, l'Association s'engage formellement à ne jamais rien ajouter, retrancher, modifier en quoi que ce soit au nombre, à la disposition et à l'aspect des peintures, sculptures, vitraux, boiseries, ferronneries, statues, autels avec leurs vases, chandeliers et autres accessoires, orgues, appareils d'éclairage, confessionnaux, chaises, bancs, cloches, dallage, tapis, crédences, 
objets mobiliers de toute espèce, etc. et d'une façon générale à tout ce qui constitue la décoration intérieure ou extérieure de l'église. Si, au cours du bail, l'Association diocésaine jugeait utile de procéder à des réparations mobilières ou immobilières, à des modifications ou additions de mobilier, elle devrait en saisir le conseil d'administration du Foyer Rémois qui serait seul juge de les autoriser ou de les refuser et qui délègue à une commission le soin de donner ou de refuser cette autorisation, comme de surveiller la réalisation des travaux autorisés. Ceux-ci devront être exécutés en respectant strictement la conception des artistes primitifs et l'esprit de leurs œuvres; les restaurations ne devront jamais s'en écarter et ne devront avoir pour but que de remettre les choses dans l'état où elles se trouvaient à l'origine.

La réalité du terrain est donc entretenue et confortée par les textes de fondation, à savoir que le mobilier de l'église Saint-Nicaise forme un tout avec le bâti puisqu'il a été conçu pour elle, dès sa construction. La protection des objets mobiliers au titre des monuments historiques est une mesure conservatoire indispensable et bénéfique. Elle ne peut néanmoins pas se suffire à elle-même. L'église Saint-Nicaise est protégée à l'heure actuelle par le fait que les bénévoles, les administrateurs du Foyer Rémois ainsi que le clergé sont sensibilisés à la valeur artistique et symbolique de son décor au sens large.

C'est pourquoi la constitution, en 2013, par l'association de l'église « Les amis de SaintNicaise du Chemin Vert", d'un inventaire sommaire avec photographies des objets mobiliers de l'église, permet d'avoir un état des lieux fiable de l'existant. Il complète la base de données Palissy pour les objets protégés. Aux objets classés au titre des monuments historiques (au nombre de 681, chaises comprises), s'ajoutent donc des objets non protégés (au nombre de 46 plus 14 poignées de porte) dont certains sont sans doute arrivés dans l'église après sa construction dans le cadre de dons faits par le commanditaire Georges Charbonneaux. Nous pouvons ainsi signaler une œuvre de Gaston La Touche, Le Portement de Croix, signée, datée de $1893^{14}$, une copie d'un tableau de Rembrandt, L'Archange Gabriel quittant la famille de Tobie (musée du Louvre), une Vierge à l'Enfant, copie dans le style de Murillo. L'huile sur toile de Maurice Denis, La Guérison de l'aveugle de Ploumanach, a été classée le 10 juin 2013 (fig. 14). La propriété de ces œuvres n'est pas encore complètement établie à ce jour, entre le Foyer Rémois et l'association diocésaine, affectataire. De même, une tapisserie donnée à l'église est aujourd'hui conservée au palais du Tau ${ }^{15}$. 


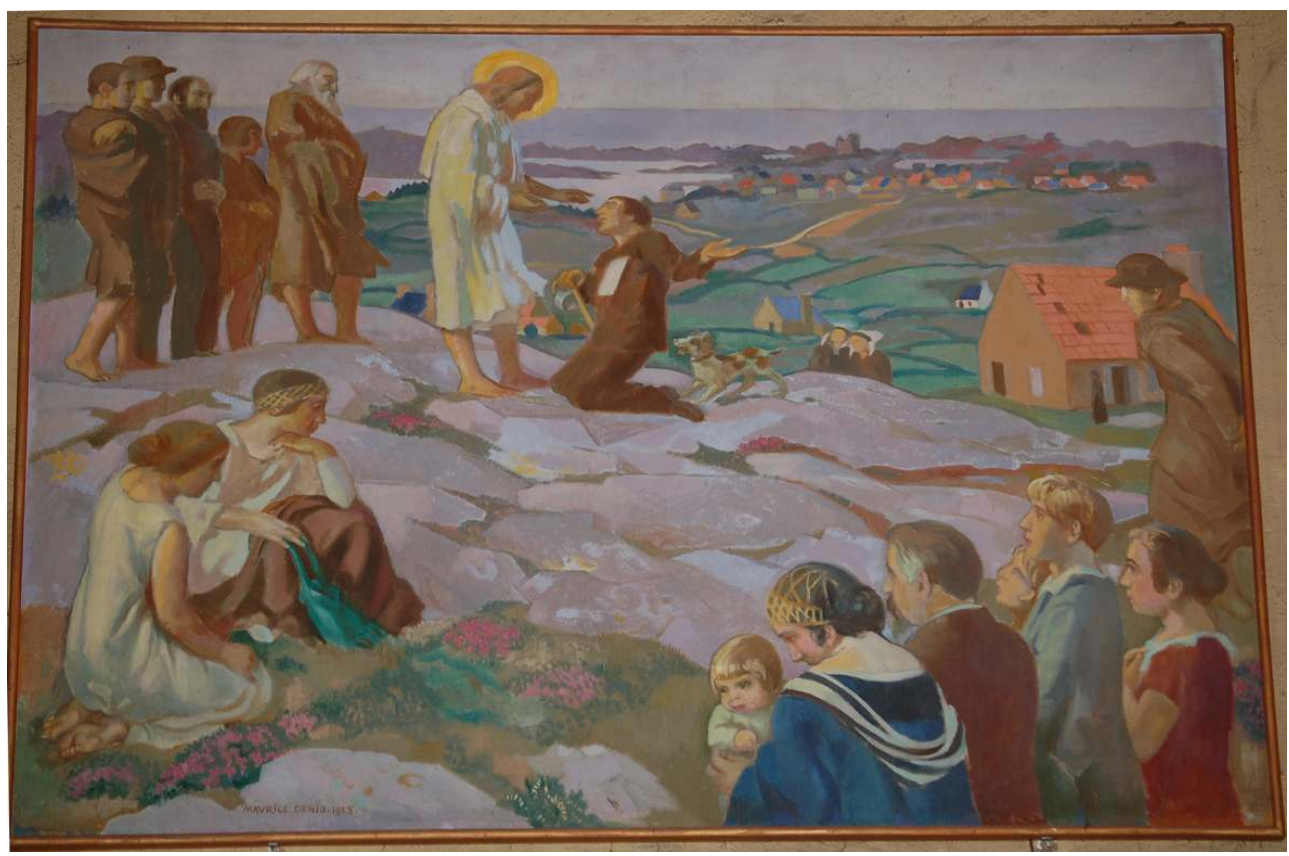

La guérison de l'aveugle de Ploumanach, Maurice Denis

Phot. CRMH Champagne-Ardenne. (c) CRMH Champagne-Ardenne.

Il est à signaler que d'ici quelques années, l'inventaire des objets mobiliers religieux de la Marne $^{16}$ intègrera cet inventaire sommaire en y incorporant des données documentaires et des sources d'archives qui renforceront encore le caractère unique de cet ensemble.

Les archives privées de Georges Charbonneaux, pour ce que la bibliographie parue à ce jour nous en révèle ${ }^{17}$, témoignent de l'importance de l'avis de l'industriel et des discussions qui eurent lieu entre lui et l'architecte Jean-Marcel Auburtin. La délicate articulation entre maître d'ouvrage et maître d'œuvre est un fait bien connu dans l'architecture pour justifier la complexité de tout projet créatif, et il revient aux historiens d'en établir a posteriori le fil linéaire et les influences respectives ${ }^{18}$. Les archives semblent néanmoins rendre compte de la sensibilité religieuse de Georges Charbonneaux qui le fait s'orienter vers les artistes des Ateliers d'Art sacré, alors dirigés par Georges Desvallières $^{19}$. Le cercle de ses amis a lui aussi joué un rôle.

L'église, son décor et ses objets mobiliers représentent l'exemple parfait d'un tout qui ne souffrirait pas d'être démembré. Chaque élément est tributaire de l'autre. Un propriétaire unique depuis l'origine, la vocation religieuse du lieu et le soutien de la population et des Amis de la cité du Chemin Vert ont favorisé le maintien in situ du mobilier. Les archives, l'inventaire et les bases de données documentaires contribuent à parachever intellectuellement cette unité et la cohérence du lieu, singulier, au sens d'unique. À l'heure actuelle, c'est le bail emphytéotique qui permet, juridiquement, de retenir la notion de responsabilité de conservation des objets mobiliers dans leur lieu d'origine sans qu'il n'y ait pour autant aucune obligation spécifiée de maintien in situ. 


\section{NOTES}

1. - POTIER, Dominique, CAUX, Yves. Reims, cité-jardin du Chemin-Vert, un siècle d'utopie raisonnée. Reims : éditions Carnet de Sentier, 2013.

2. - 7 février 1923, début des travaux ; 8 juin 1934, consécration. Le décor et les objets mobiliers de l'église furent réalisés entre 1924 et 1934.

3. - CHATELIN, Patrick. "L'église Saint-Nicaise de la cité du Chemin Vert à Reims ». Le Point riche, bulletin de l'association «Les amis de Louis Mazetier » et de l'art sacré du Xx $x^{\mathrm{e}}$ siècle, $\mathrm{n}^{\circ}$ 9, juin 2011, p. 7-24.

4. - L'église a été classée en totalité par arrêté du 13 décembre 2002, les objets mobiliers par arrêté du 10 juin et du 19 septembre 2013.

5. - Outre la sécurisation des accès, un inventaire sommaire des objets mobiliers dans leur ensemble a été entrepris par l'association, sur les conseils de la conservatrice des Monuments historiques. Une couverture photographique a été réalisée par M. Dominique Potier, photographies disponibles pour la base Palissy du ministère de la Culture ou pour les publications, avec copyright.

6. - Les artistes qui ont contribué au décor de l'église Saint-Nicaise sont cités dans l'article de Patrick Chatelin, «L'église Saint-Nicaise de la cité du Chemin Vert à Reims », op. cit.

7. - Relèvent de la catégorie des « objets mobiliers» telle que définie par le livre VI du Code du patrimoine les biens "meubles par nature " au sens de l'article 528 du Code civil et les biens « immeubles par destination » au sens des articles 524 et 525 du même Code (extrait de la note du ministère de la Culture du 28 novembre 2012 sur la protection des objets mobiliers).

8. - Au nombre de 576 !

9. - En fer forgé, par la maison Berger-Bogard. Dessin de Jean-Marcel Auburtin.

10. - Cet ostensoir fut redécouvert au moment de l'inventaire et mériterait d'être également protégé au titre des monuments historiques.

11. - Notamment à Douvres-la Délivrande (Calvados), en Normandie.

12. - Dépose au printemps-été 2014. Rapport de visite, Claudine Loisel et Annick Texier, LRMH (Laboratoire de recherche sur les monuments historiques), 16 mai 2014.

13. - Acte du 2 juin 1927 par devant Maîtres Humbert et Marret entre le Foyer Rémois et l'association diocésaine de Reims représentée par le cardinal Luçon (archives du Foyer Rémois).

14. - Prêté au musée des Avelines de Saint-Cloud (Hauts-de-Seine) pour l'exposition monographique « Gaston La Touche - Les fantaisies d'un peintre de la Belle Époque », 16 octobre 2014 - $1^{\mathrm{er}}$ mars 2015.

15. - Information donnée par le curé de la paroisse et le Foyer Rémois.

16. - Cet inventaire clôturera la campagne d'inventaire informatisé des objets mobiliers religieux des quatre départements de la région, lancée en 2003 par la conservation régionale des Monuments historiques de Champagne-Ardenne ; celle-ci débuta par l'Aube, puis fut suivie par la Haute-Marne et enfin, en 2014, par les Ardennes. Ces bases sont co-financées par l'État, la région et le conseil départemental et seront désormais accessibles sur l'outil de l'inventaire régional «Gertrude » et, à terme, sur les bases de données nationales Palissy et Mémoire.

17. - Se reporter à l'article de Patrick Chatelin et à la bibliographie, p. 24.

18. - Voir sur le site d'In Situ les numéros 11 et 12 : «Le patrimoine religieux des $\mathrm{XIX}^{\mathrm{e}}$ et $\mathrm{XX}^{\mathrm{e}}$ siècles » http://insitu.revues.org/4188 et http://insitu.revues.org/4657.

19. - Georges Desvallières (1861-1950) est un artiste engagé qui a travaillé au renouvellement de l'art religieux dès son entrée à la Société de Saint-Jean en 1906. Il fut profondément marqué par 
la Première Guerre mondiale au cours de laquelle il perdit l'un de ses fils. Il se consacra alors à la peinture religieuse et créa les Ateliers d'Art Sacré avec Maurice Denis en 1919. Une exposition monographique lui est dédié au Petit-Palais en 2016.

\section{RÉSUMÉS}

L'église Saint-Nicaise de Reims, construite par l'architecte Jean-Marcel Auburtin et décorée par de nombreux artistes, dont Maurice Denis, entre 1925 et 1933, est gérée par un bail emphytéotique entre le propriétaire, office d'habitation à loyers modérés, et l'association diocésaine. Cette disposition permet la mise en valeur et le maintien in situ de l'ensemble des objets mobiliers, conçus spécifiquement pour l'église et classés majoritairement au titre des monuments historiques.

The Saint-Nicaise Church of Rheims was designed by the architect Jean-Marcel Auburtin in 1925 and decorated by several artists, including Maurice Denis. The chruch is managed today under the terms of an emphyteutic lease between the owner, a local council housing organisation, and the ssociation of the diocese. This provision allows for the interpretation and conservation in situ of all the movable objects, specifically designed for the church and protected, for the most part, as historic monuments.

\section{INDEX}

Keywords : church furniture, Jacques-Marcel Auburtin, Le Foyer Rémois, Georges Charbonneaux, decorative art, computerized inventory

Mots-clés : mobilier religieux, Jacques-Marcel Auburtin, le Foyer rémois, Georges

Charbonneaux, art décoratif, inventaire informatisé

\section{AUTEUR}

\section{ISABELLE MORIN LOUTREL}

Conservatrice du patrimoine chargée des collections d'architecture du musée d'Orsay isabelle.morin-loutrel@musee-orsay.fr 